\title{
Synchronicity belies simplicity in short-term memory
}

Most of us draw on our visual recognition and short-term memory abilities many times a day and with relatively little fanfare, but these seemingly mundane activities have rather complex underpinnings within our brains. The tasks involve different, non-adjacent brain regions: short-term memory formation occurs in the lateral prefrontal cortex (PFC), and visual information processing primarily takes place in the occipital lobe, in the back part of the brain's cerebral cortex. It is believed that these brain regions must interact during tasks requiring the use of shortterm memories for visual recognition, but the nature and mechanism of their interaction is not well understood.

Stefanie Liebe and Gregor Rainer (Max Planck Institute for Biological Cybernetics, Tuebingen, Germany) led a study to investigate the potential interaction between the brain areas. They examined neural communication between the PFC and visual area V4 during short-term memory formation and recall in two adult male macaques (Macaca mulatta) performing a visual recognition task. The monkeys were shown a sample image from a set including birds, flowers, butterflies and monkeys in their natural surroundings. Then, after a brief delay, they were shown a test image that was either identical to or different from the sample. The monkeys were given juice when they correctly indicated whether or not the test image matched the sample.

Liebe and Rainer's group found that electrical oscillations in the PFC and V4 became synchronized during memory formation, particularly in a range of frequencies $(3-9 \mathrm{~Hz})$ called the theta band (Nat. Neurosci. doi:10.1038/nn.3038; published online 29 January 2012). "It is as if you have two revolving doors in each of the two areas. During working memory, they get in sync, thereby allowing information to pass through them much more efficiently than if they were out of sync," explained Liebe in a press release.

Stronger synchronization between the two regions resulted in better recall performance in the macaques, a finding that

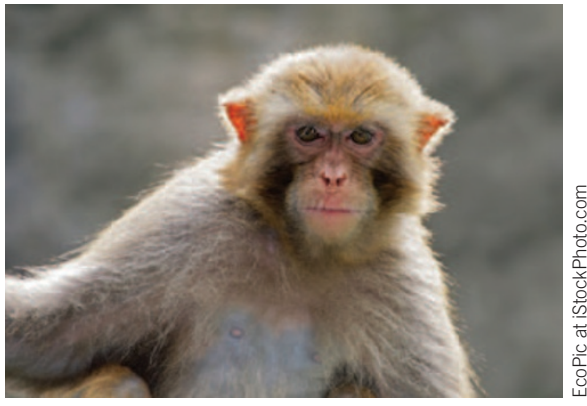

demonstrates how changes in the electrical activity of the brain can contribute directly to behavior. The researchers conclude that theta-band synchronization may enable short-term visual memory by coordinating communication between the distant PFC and V4 regions.

Interaction between distant regions of the brain is crucial in many cognitive functions in addition to visual recognition. More research is needed to understand how these interactions are established and maintained, as well as how they participate in the transmission of information.

Monica Harrington

\section{KEY TO GROWING BLOOD-FORMING STEM CELLS DISCOVERED}

Billions of new blood cells are produced every day from the hematopoietic stem cells (HSCs) in our bone marrow. Although scientists have determined how to make stem cells for other specific types of tissues in the lab, efforts to create these bloodforming stem cells in the lab have so far not been successful. One of the challenges is that the environment necessary for the stem cells' growth is poorly understood.

Stem cells are maintained in specialized microenvironments called niches, in which other cells secrete proteins, such as stem cell factor (SCF), that support stem cell maintenance. Most HSCs localize along the blood vessels in bone marrow. A new study reveals that endothelial cells and perivascular stromal cells in bone marrow are responsible for secreting the SCF that maintains HSCs in the body.

By replacing the gene Scf with one that encodes green fluorescent protein (GFP) in mice, Sean J. Morrison of the Howard Hughes Medical Institute at University of Texas Southwestern Medical Center (Dallas, TX) and collaborators at University of Michigan (Ann Arbor) and Cold Spring Harbor Laboratory (Cold Spring Harbor, NY) could systematically identify any cells that glowed green as ones involved in producing the crucial SCF. In this way, endothelial cells and stromal cells were identified as the major sources of SCF in bone marrow (Nature 481, 457-462; 2012). Furthermore, genetically preventing production of SCF from these cells in mice caused a severe reduction in blood cell number. Interestingly, the researchers also found that Scf expression by hematopoietic cells was not required for HSC maintenance in adult bone marrow.

Although endothelial cells and perivascular stromal cells were identified as functionally important components of the HSC niche, further research is needed to identify other signals from these cells that may promote the growth of blood-producing stem cells. This will allow scientists to replicate the necessary conditions to grow HSCs in the laboratory. The research also has implications for transplanting bone marrow and umbilical cord blood in humans: if blood-forming stem cells can be grown in the lab, they could then be transplanted prior to surgery to make the procedures safer and more effective.

Kara Rosania 